\title{
Multi-resolution Modeling In Application Of Power Supply System Compatibility On More Electric Aircraft
}

\author{
Lirong Sun ${ }^{1, a}$, Zheng $\mathrm{Cao}^{2, \mathrm{~b}}$, Liang $\mathrm{Liu}^{3, \mathrm{c}}$ and Yuanjun Zhou ${ }^{4, \mathrm{~d}}$ \\ ${ }^{1} 601$ Aricraft Design Institute, Shenyang, China \\ ${ }^{2}$ Department of Electrical Energineering, Beihang University, China \\ ${ }^{3} 601$ Aricraft Design Institute, Shenyang, China \\ ${ }^{4}$ Department of Electrical Energineering, Beihang University, China \\ asunlrong@163.com, bcaozheng1203158@163.com, 'liuliang10.16@163.com, \\ zzhouyuanjun@buaa.edu.cn
}

Keywords: multi-resolution modeling, power system compatibility, more electric aircraft

Abstract. In order of saving energy and improving the performance of the airborne systems, modern aircraft increasingly uses electrical energy to replace the current hydraulic, pneumatic energy on the plane. The goal of more electric aircraft is that electric drive equipments replace mechanical, hydraulic and pneumatic equipments directly driven by the original engine to obtain high performance and high efficiency. The increase of electrical loads leads to cause power supply system compatibility problems. The concerned issue is power interference by power electronic loads, including electric actuator which is the most typical interference load.

The common research method of solving power system compatibility is integrated simulation and analysis which include power, cables, electric equipments. Due to diversity of system compatibility issues, it is difficult to realize the large-scale integrated simulation. This paper applies multi-resolution modeling in power supply system compatibility simulation, and describes conductive electromagnetic interference, power stability interference and disturbance of dynamic load power which is electric actuator as typical electrical equipment.

\section{Introduction}

Nowadays, as electronic loads of more electric aircraft increase, the compatibility of power supply system is focused. The 270V DC aircraft power supply system structure as shown in Fig. 1, DC power supplies for DC bus, and different loads connected on the bus-bar, of which is a significant proportion of power electronic devices.

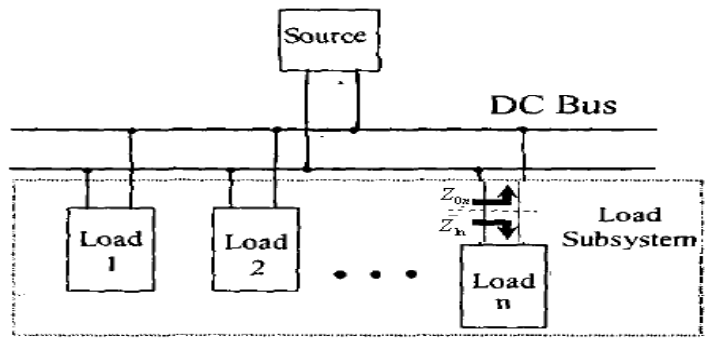

Fig. 1 The structure of more electric aircraft power supply system

In power supply system of more electric aircraft, power electronic loads as the interference sources impact on power quality. In system simulation, load models are described as the form of impedance. In Fig. 1, the load $\mathrm{n}$ is $\mathrm{Z}_{\mathrm{n}}$ for load subsystem, but it is $\mathrm{Z}_{0 \mathrm{~N}}$ for source. Load impedance $Z_{\mathrm{n}}$ should be able to accurately show characteristics of sources interference, due to complicated interference signal types, it needs a suitable model to be described.

According to load characteristic analysis of electrical equipment in more electric aircraft system, the compatibility of power supply system exists the following problems: 
The problem of conductive electromagnetic interference.

Due to the load characteristic of chopper, for the power conductive electro-magnetic interference, EMI filter should be designed. Although the design of EMI filter has certain theory and method, but the extent of power disturbances is associated with the power structure which includes distribution parameters of cable, characteristics of power supply and performance of other electrical equipments.

The problem of power stability

There are constant power loads which are negative impedance characteristics for power, distribution capacitance and inductance. All of these should influence power stability. Also, solving the stability of single equipment has specific theory and method, but the stability associated with power structure which includes the parameters of power supply and electric equipments.

The problem of dynamic load disturbance

The characteristics of dynamic loads are large transient power, short work and power disturbance. The electric actuator of more electric aircraft is the most typical dynamic load. The pulse loads repeatedly impact on power, and power disturbance influence depends on characteristics of power supply.

Obviously, because the compatibility of power supply system is related to characteristics of power, distribution parameters of power and load characteristics of electric equipments, adopting integration simulation of power supply system is an effective method.

\section{Models of power supply system compatibility on more electric aircraft}

With the improvement of computer performance, high-resolution models seem to fully meet the simulation needs. However, the pursuit of high resolution models often increases computational load of computer and cannot solve problems. Different problems need different resolutions, and different resolution models have different uses.

The characteristics of multi-resolution models. Multi-resolution modeling is facing the same problem space, considering a number of different resolution levels, and establishing a single model or a set of models between all levels to achieve seamless docking.

High-resolution models can be used to study the details of system, which is suitable for study of specific phenomena, simulation reality, low resolution model check, and use of high-resolution data and knowledge. Low-resolution models can grasp overall features of system from the macro, which are applicable to system analysis, policy analysis, exploratory analysis, rapid prototyping, and use of low-resolution data and knowledge.

There are two main types of multi-resolution modeling: the entity oriented multi-resolution modeling and the parameter oriented multi-resolution modeling. The parameter can not only be a physical properties but also be a performance index of the evaluation function. The parameter oriented multi-resolution modeling is a hierarchical decomposition and polymerization method based on the parameters. The parameters can change according to levels, namely high parameter can be input directly, also can be obtained by the underlying operational parameters.

This paper adopts multi-resolution modeling to describe problems of aircraft power supply system compatibility, then simplify model structure by an aggregation and decomposition method to realize conveniently large-scale integrated simulation.

Admittance models of electrical equipments. Electrical equipments shown in circuit of Fig. 1, impedance can be adopted by the concept of two-port network and described as power voltage and load current. For the i-th dynamic impedance of electrical equipment, it can be expressed respectively in the time and frequency domain.

$$
Z_{i}(t)=\frac{U(t)}{I_{i}(t)} \quad Z_{i}(s)=\frac{U(s)}{I_{i}(s)} .
$$


In simulation, electrical equipments are considered as loads. When there is the input power voltage, the impedance determines input current. Therefore, in the description of analytical formula, adopting admittance model is more accurate.

$$
Y_{i}(t)=\frac{I_{i}(t)}{U(t)}=\frac{1}{Z_{i}(t)}, \quad Y_{i}(s)=\frac{I_{i}(s)}{U(s)}=\frac{1}{Z_{i}(s)} .
$$

The electrical equipments in power supply system, on the other hand, are in parallel on power, adopting admittance model is easier to implement impedance characteristics of multiple equipments.

Frequency characteristics of load admittance models. In the study of power supply system compatibility, simulation models of electrical equipments should be able to accurately show interference characteristics of power. The interference signals of electrical equipments on aircraft can be distinguished by frequency band.

(1) The conductive electromagnetic interference on aircraft power is mainly produced by power electronic devices adopted PWM signal control, the frequency of electromagnetic compatibility jamming signal is greater than 10 to $20 \mathrm{kHz}$.

(2) The main factor of power stability influence is the constant power load, and the resonance frequency of instability is produced by the parameters of EMI filter. The resonance frequency is usually 1 to $10 \mathrm{kHz}$.

(3)The power disturbance of dynamic loads is formed due to the motion characteristics of servo control system, the disturbance frequency is lower, typically below $100 \mathrm{~Hz}$.

Therefore, in electrical equipment modeling, the frequency band can be used as multi-resolution parameters, and multi-resolution models can be set up for solving different problems.

\section{The multi-resolution model of electromechanical actuator}

The electromechanical actuator of more electric aircraft is considered to be the biggest influence on power quality, with all the characteristics of electromagnetic interference, damage stability and high power disturbance for power system.

The electromechanical actuator control system. The electromechanical actuator is an aircraft rudder driven device, and the principle is shown in Fig. 2. The system is three closed-loop control system of brushless dc motor, which includes position, speed and current closed-loop control, and EMI filter with LC structure in power input.

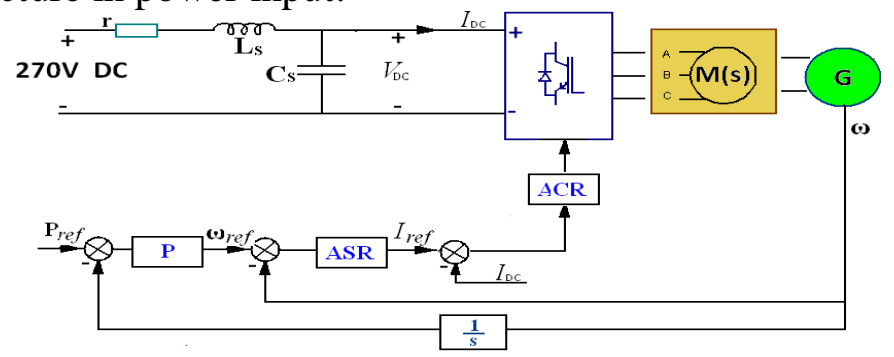

Fig. 2 The principle of electromechanical actuator

For electromechanical actuator structure, the control system is a high order and nonlinear mathematical model. The basic equation is:

$$
\begin{aligned}
& u_{d}=L_{a} \frac{d i_{d}}{d t}+R_{a} i_{d}+K_{e} \omega_{m} \\
& J \frac{d \omega_{m}}{d t}=K_{T} i_{a}-B_{\omega} \omega_{m}-K_{\theta} \theta_{a}-T_{f} \\
& \frac{d \theta_{a}}{d t}=K_{\omega} \omega_{m}
\end{aligned}
$$


Where $i_{d}$ is dc bus current of inverter, $u_{d}$ is dc bus voltage, $\omega_{n}$ is motor speed, $\theta_{a}$ is rudder location. Parameters $L_{a}$ and $R_{a}$ are respectively the equivalent inductance and resistance of motor armature winding, $K_{\varepsilon}$ and $K_{T}$ are motor electric potential and torque coefficient, $J$ is inertia of motor system, $\mathrm{B} \omega$ is damping coefficient, $\mathrm{K}_{\theta}$ is hinge moment coefficient of actuator, $\mathrm{T}_{\mathrm{f}}$ is machinery friction torque, and $\mathrm{K}_{\omega}$ is reduction ratio of gear. Among them, $\mathrm{u}_{\mathrm{d}}$ is the PWM control signal, which is a nonlinear function.

In closed loop control system, current and speed regulators contain integral element, thus it should add differential relationship.

$$
\frac{d e_{i}}{d t}=i_{d}^{*}-i_{d}, \frac{d e_{\omega}}{d t}=\omega_{m}^{*}-\omega_{m}, \frac{d e_{\theta}}{d t}=\theta_{a}^{*}-\theta_{a} .
$$

The state space of mathematical model is:

$$
\frac{d}{d t}\left[\begin{array}{l}
i_{d} \\
\omega_{m} \\
\theta_{a} \\
e_{i} \\
e_{\omega} \\
e_{\theta}
\end{array}\right]=\left[\begin{array}{cccccc}
-\frac{R_{a}}{L_{a}} & -\frac{K_{e}}{L_{a}} & 0 & 0 & 0 & 0 \\
\frac{K_{T}}{J} & -\frac{B_{\omega}}{J} & -\frac{K_{\theta}}{J} & 0 & 0 & 0 \\
0 & K_{\omega} & 0 & 0 & 0 & 0 \\
-1 & 0 & 0 & 0 & 0 & 0 \\
0 & -1 & 0 & 0 & 0 & 0 \\
0 & 0 & -1 & 0 & 0 & 0
\end{array}\right] \cdot\left[\begin{array}{l}
i_{d} \\
\omega_{m} \\
\theta_{a} \\
e_{i} \\
e_{\omega} \\
e_{\theta}
\end{array}\right]+\left[\begin{array}{l}
\frac{1}{L_{a}} \\
0 \\
0 \\
0 \\
0 \\
0
\end{array}\right] u_{d}+\left[\begin{array}{l}
0 \\
-\frac{T_{f}}{J} \\
0 \\
i_{d}^{*} \\
\omega_{m}^{*} \\
\theta_{a}^{*}
\end{array}\right]
$$

Considering closed loop control, the equation is:

$$
u_{d}=k_{1} i_{d}+k_{2} \omega_{m}+k_{3} \theta_{a}+k_{4} e_{i}+k_{5} e_{\omega}+k_{6} e_{\theta}
$$

The state equation of closed loop control system is:

$$
\frac{d}{d t}\left[\begin{array}{l}
i_{d} \\
\omega_{m} \\
\theta_{a} \\
e_{i} \\
e_{\omega} \\
e_{\theta}
\end{array}\right]=\left[\begin{array}{cccccc}
\frac{k_{1}-R_{a}}{L_{a}} & \frac{k_{2}-K_{e}}{L_{a}} & \frac{k_{3}}{L_{a}} & \frac{k_{4}}{L_{a}} & \frac{k_{5}}{L_{a}} & \frac{k_{6}}{L_{a}} \\
\frac{K_{T}}{J} & -\frac{B_{\omega}}{J} & -\frac{K_{\theta}}{J} & 0 & 0 & 0 \\
0 & K_{\omega} & 0 & 0 & 0 & 0 \\
-1 & 0 & 0 & 0 & 0 & 0 \\
0 & -1 & 0 & 0 & 0 & 0 \\
0 & 0 & -1 & 0 & 0 & 0
\end{array}\right] \cdot\left[\begin{array}{l}
i_{d} \\
\omega_{m} \\
\theta_{a} \\
e_{i} \\
e_{\omega} \\
e_{\theta}
\end{array}\right]+\left[\begin{array}{l}
0 \\
-\frac{T_{f}}{J} \\
0 \\
i_{d}^{*} \\
\omega_{m}^{*} \\
\theta_{a}^{*}
\end{array}\right]
$$

The position output of actuator is:

$$
y=\theta_{a}
$$

The dynamic power model. The input power of electromechanical actuator could change according to motion state of control system, thus the dynamic power model depends on the servo control system model.

The control system model should be selected in less than $100 \mathrm{~Hz}$ low frequency band, which belongs to the low-resolution model. The average voltage of control signal uses mean method, and the relationship between power voltage is: 
$u_{d}=K_{V} V_{d c}, i_{d}=\frac{i_{d c}}{K_{V}}$

where $K_{V}$ is duty of PWM signal.

When power voltage has fluctuations, namely

$u_{d}=K_{V} V_{d c}+\Delta V_{d c}$

Ignoring of disturbance influence $\mathrm{T}_{\mathrm{f}}, \mathrm{i}_{\mathrm{d}}{ }^{*}$ and $\omega_{\mathrm{m}}{ }^{*}$, the admittance model which reflects impacts of electromechanical actuator on power is:

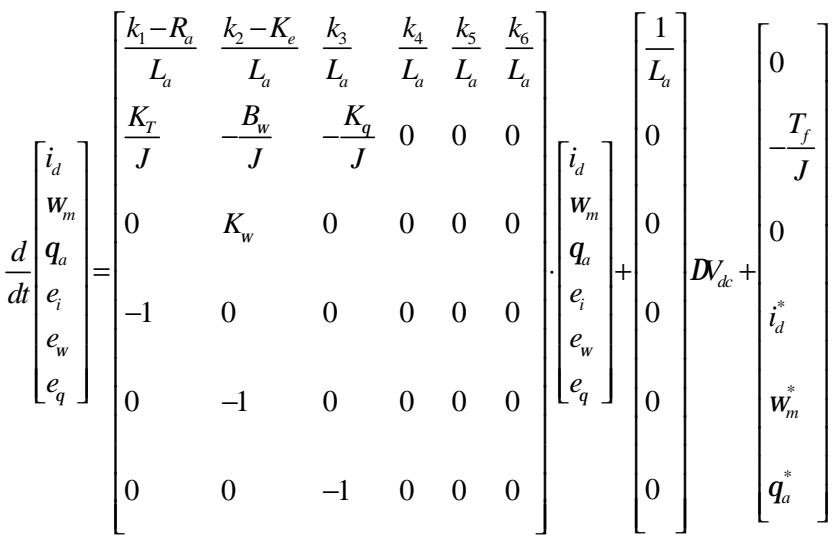

$$
\begin{aligned}
& y=i_{d c}=K_{v} i_{d}
\end{aligned}
$$

The equation (11) and (12) are written in admittance model, and equation (13) is admittance model of position control system.

$$
Y_{A}(s)=\frac{I_{d c}(s)}{\Delta V_{d c}(s)}
$$

The load stability model. The cut-off frequency of EMI filter in power input is $7 \mathrm{kHz}$, and load stability model can be established in 1 to $10 \mathrm{kHz}$ frequency band. For servo control system of less than $100 \mathrm{~Hz}$ frequency domain, the model can be described using equivalent resistance. Due to constant power load, it is often expressed by $-\mathrm{R}_{\mathrm{L}}$, the impedance is:

$$
\left|Z_{A}(s)\right|_{s=j \omega}=\left|\frac{1}{Y_{A}(s)}\right|_{s=j \omega}=\left|\frac{\Delta V_{d c}(s)}{I_{d c}(s)}\right|_{s=j \omega}
$$

Considering the most serious stability situation, the equivalent impedance is :

$$
\left|R_{L}\right|=\left|Z_{A}(j \omega)\right|_{\max }=\left|\frac{1}{Y_{A}(j \omega)}\right|_{\max }=\left|\frac{\Delta V_{d c}(j \omega)}{I_{d c}(j \omega)}\right|_{\text {max }}
$$

The load stability model is shown in Fig. 3. 


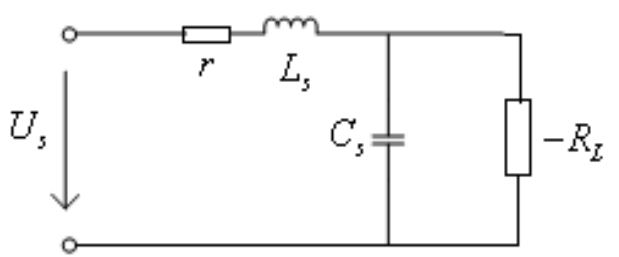

Fig. 3 The load stability model

If do not consider power internal resistance, admittance model is obtained.

$$
Y_{B}(s)=\frac{I_{s}(s)}{U_{s}(s)}=\frac{C_{s} R_{L} s-1}{C_{s} L_{s} R_{L} s^{2}-L_{s} s+R_{L}}
$$

Obviously this is an unstable system, the system stability depends on power parameters. Considering power internal resistance, the admittance model of closed-loop control chopper is:

$$
Y_{B}(s)=\frac{I_{s}(s)}{U_{s}(s)}=\frac{C_{s} R_{L} s-1}{C_{s} L_{s} R_{L} s^{2}+\left(C_{s} R_{L} r-L_{s}\right) s+\left(R_{L}-r\right)}
$$

Visibly, stability condition of electric equipment is:

$$
R_{L}>r, r R_{L} C_{s}>L_{s} \text {. }
$$

If power internal resistance and load power are constant, increasing filter capacitance and reducing filter inductance make power system stability.

The electromagnetic compatibility model. The chopping frequency of inverter is selected with more than $20 \mathrm{kHz}$, and electromagnetic interference frequency band should be the same. Therefore, electromagnetic interference model should be established in more than $20 \mathrm{kHz}$ high frequency band, and structure is shown in Fig. 4. The actuator power, namely the filter power output, is constant and the power is stable. The inverter pulse current is equivalent to a pulse load.

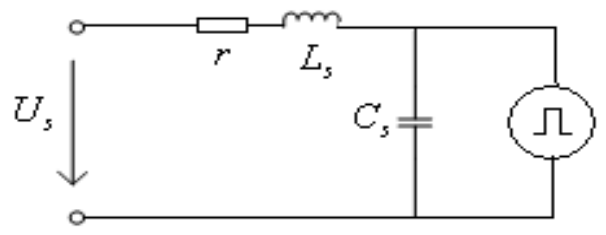

Fig. 4 The electromagnetic compatibility model

If the pulse load change is described as resistance and pulse cycle is $T_{p}$, for pulse resistance is:

$$
R_{p}(t)= \begin{cases}R_{L} & 0 \leq t<t_{1} \\ \infty & t_{1} \leq t<T_{p}\end{cases}
$$

The admittance model is obtained.

$$
Y_{C}(s)=\frac{I_{s}(s)}{U_{s}(s)}=\frac{C_{s} R_{p} s+1}{C_{s} L_{s} R_{p} s^{2}+\left(C_{s} R_{p} r+L_{s}\right) s+\left(R_{p}+r\right)}
$$

Apparently, nonlinear resistance $\mathrm{R}_{\mathrm{p}}$ in equation (20) leads to complex admittance algorithm. If pulse load is described using pulse current, namely 


$$
I_{p}(t)= \begin{cases}I_{L} & 0 \leq t<t_{1} \\ 0 & t_{1} \leq t<T_{p}\end{cases}
$$

The power input current is obtained.

$$
\begin{aligned}
& I_{s}=I_{c}+I_{p}=C_{s} s\left[U_{s}-I_{s}\left(r+L_{s} s\right)\right]+I_{p} \\
& I_{s}=\frac{C_{s} s}{\left(1+r C_{s} s+L_{s} C_{s} s^{2}\right)} U_{s}+\frac{1}{\left(1+r C_{s} s+L_{s} C_{s} s^{2}\right)} I_{p}=I_{s 1}+I_{s 2}
\end{aligned}
$$

In equation (23), the first item on the right is surge current cause by power voltage change, the second is power current caused by pulse current. Considering power voltage mutation, the admittance model further is:

$$
Y_{C}(s)=\frac{I_{s 1}}{U_{s}}=\frac{C_{s} s}{\left(1+r C_{s} s+L_{s} C_{s} s^{2}\right)}
$$

The admittance model of pulse current is obtained.

$$
Y_{D}(s)=\frac{I_{s 2}}{U_{s}}=\frac{1}{\left(1+r C_{s} s+L_{s} C_{s} s^{2}\right)} \frac{I_{p}}{U_{s}}
$$

\section{The results of load characteristic simulation}

Adopting the admittance model of electromechanical actuator in different frequency bands, the power of electromechanical actuator is $5 \mathrm{~kW}$.

The dynamic power simulation. According to equation (11) and (12), the rudder movement angle is given $25^{\circ}$. The results are shown in Fig. 5. and show that a large power created when motor starts. The peak value is $8.2 \mathrm{~kW}$. When rudder moves to a given position, due to the hinge moment achieved to maximum, the electric power demand is about $6.3 \mathrm{~kW}$.

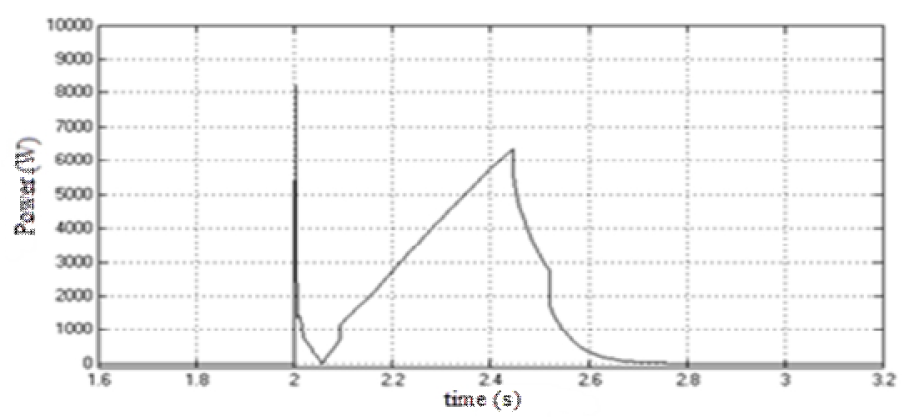

Fig. 5 The simulation results of dynamic power

The power stability simulation. Using equation (17) to simulate the power stability model, results are shown in Fig. 6. When the Cs/Ls ratio is 0.37 , the system is in critical stable state, and the current appears with equal amplitude turbulence, as shown in figure (a). When the Cs/Ls ratio is 0.88 , the system satisfies stability condition, and stabilizes after a brief wobble, as shown in figure (b). 


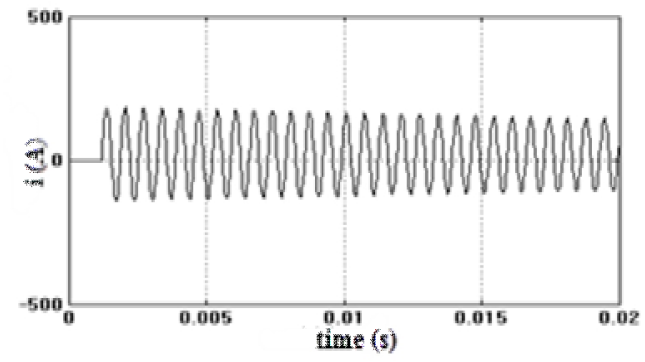

(a) $\mathrm{Cs} / \mathrm{Ls}=0.37$

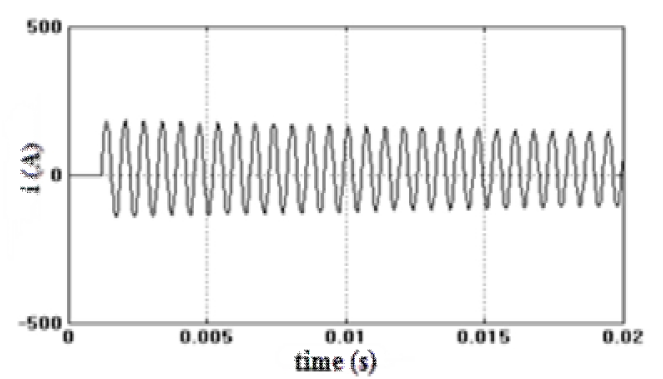

(b) $\mathrm{Cs} / \mathrm{Ls}=0.88$

Fig. 6 The curve of power input admittance model

The electromagnetic compatibility characteristic simulation. In equation (25), chopper frequency of inverter is $10 \mathrm{kHz}$, power is $9.5 \mathrm{~kW}$, and cut-off frequency of filter is $3 \mathrm{kHz}$. Simulation waveforms are shown in Fig. 7. There is no filter current waveform in the above figure with pulse current amplitude of $70 \mathrm{~A}$, and the below figure is the current waveform including filter with pulse current amplitude of $4 \mathrm{~A}$.

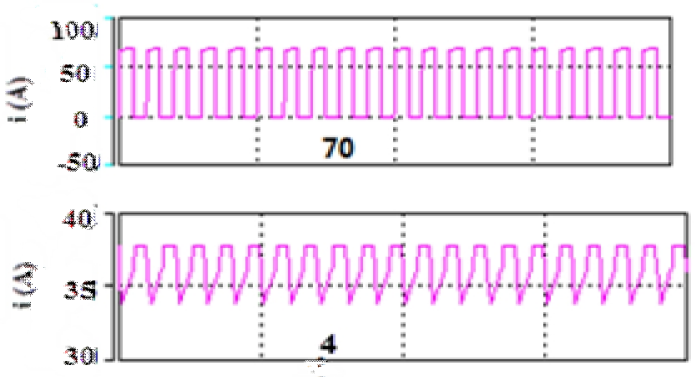

Fig. 7 The current waveforms of EMI filter

\section{Conclusion}

With multi-resolution parameters based on frequency response, the multi-resolution modeling of electrical equipments could accurately describe the characteristics of interference sources. The application makes models to be simple structure, and conveniently realizes the integration simulation of power supply system compatibility.

\section{Acknowledgements}

This work was financially supported by Aeronautical Science Foundation (2014ZC01002).

\section{References}

[1] C.G.Cassandras, C.G.Panayiotou, G.Diehl, W-B.Gong, Z.Liu, and C.Zon. Clustering methods for multi-resolution simulation modeling. Enabling Technology for Simulation Science IV, Proceedings of SPIE, 2000, 4026: 37 48.

[2] Satoshi Sekine, Makoto Kanou. An Investigation of Advanced Technique for MRM (multi-resolution models). Proceedings of the 10th CGF\&BR Conference, 2001.5.

[3] Lee K. and Fishwick P.A. ACM Transactions on Modeling and Computer Simulation. Vol.9, No.2.pp.141-170.1999.

[4] J. Weimer. Electrical power technology for the more electric aircraft. Conference Proceedings of IEEE DASC, vol. 3, pp. 445-450, 1993.

[5] K.J. Karimi. The role of power electronics in more-electric airplanes(MEA). IEEE Workshop on Computers in Power Electronics, July 2006. 